Literature Review

\title{
Anesthetic Considerations and Perioperative Management of Spinal Cord Stimulators: Literature Review and Initial Recommendations
}

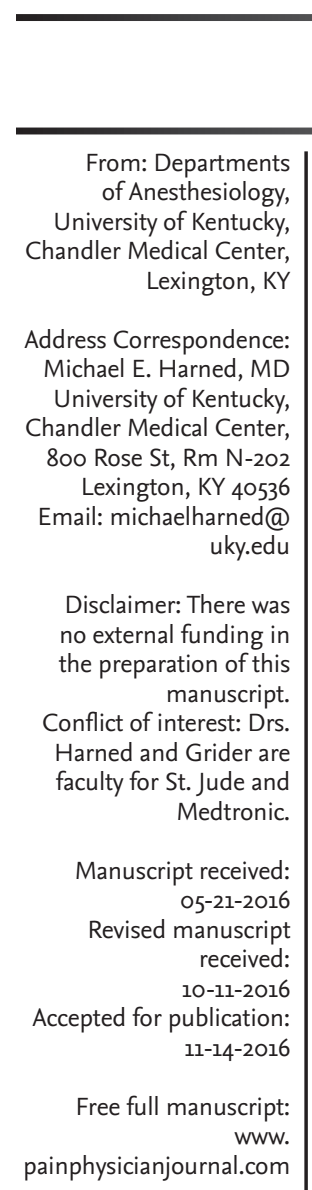

Michael E. Harned, MD, Brandon Gish, MD, Allison Zuelzer, MD, and

Jay S. Grider, DO, PhD equipment and pose significant risk to patient and device. management of SCS in the perioperative period and to begin to develop recommendations. locations. and Google Scholar was performed. Websites of SCS manufactures were reviewed.

Background: Patients with implanted spinal cord stimulators (SCS) present to the anesthesia care team for management at many different points along the care continuum. Currently, the literature is sparse on the perioperative management. What is available is confusing; monopolar electrocautery is contraindicated but often used, full body magnetic resonance imaging (MRI) is safe with particular systems but with other manufactures only head and specific extremities exams are safe. Moreover, there are anesthetizing locations outside of the operating room where implanted SCS can interact with surrounding medical

Objectives: The objective of this review is to present relevant known literature about the safe

Study Design: A review of current literature and each manufacturers' labeling was performed to assess risk of interference and patient harm between SCS and technology used in and around typical anesthetizing

Methods: A systematic search of the literature was conducted in accordance with the Preferred Reporting Items for Systematic Reviews and Meta-Analysis (PRISMA) statement. A computerized search was conducted for English articles in print up to April 2016 via PubMed www.ncbi.nlm.nih.gov/pubmed; EMBASE www.embase. com; and Cochrane Library www.thecochranelibrary.com. Search terms included "spinal cord stimulator AND MRI," "spinal cord stimulator AND ECG," "spinal cord stimulator AND implanted cardiac device," "spinal cord stimulator AND electrocautery," and "spinal cord stimulator AND obstetrics." In addition, a search of Google

Results: Generalized recommendations include turning the amplitude of the SCS to the lowest possible setting and turning off prior to any procedure. Monopolar electrocautery is contraindicated but is still often utilized; placing grounding pads as far away from the device can reduce the risk to device and patient. Bipolar cautery is favored. Implanted cardiac devices can interfere with SCS, but risks can be minimized. Neuraxial anesthesia can be attempted in a patient with implanted SCS, provided the device is not in the expected path. MRI labeling differences present the biggest difference among SCS manufactures. Medtronic's SureScan SCS, Boston Scientific's Precision system, St. Jude's Proclaim, and Stimwave's Freedome SCS are full body MRI compatible under specific conditions, while other manufacturers have labeling that restricts exams of the trunk and certain extremities.

Limitations: This review was intended to be a comprehensive, cumulative review of recommendations for perioperative SCS management; however, the limitations of a review of this nature is the complete reliance on previously published research and the availability of these studies using the methods outlined.

Conclusions: SCS is being used earlier in the treatment algorithm for patients with chronic pain. The anesthesia care team needs working knowledge of where the device resides in the neuraxial space and what risks different medical technologies pose to the patient and device. This understanding will lead to appropriate perioperative management which can reduce risk and improve patient outcomes.

Key words: Spinal cord stimulation, perioperative management, MRI, anesthetic considerations,

CT scan, interventional pain management

Pain Physician 2017; 20:319-329 
S pinal cord stimulation (SCS) has become increasingly important in the management of chronic pain conditions including chronic back and leg pain, complex regional pain syndrome, refractory cardiovascular conditions, and painful peripheral neuropathy (1). As a result, it will become increasingly common for the anesthesia care team (ACT) to provide care for patients with implanted neuromodulation devices (2). Moreover, chronic pain patients are known to have increased rates of medical service utilization (3). Recommendations regarding perioperative management of targeted drug delivery systems have been in the literature since 2008, but recommendations regarding SCS have been slow to materialize (4). With the increasing complexity of current and future SCS and the potential for adverse device and patient outcomes, the $A C T$ is required to have more than just a superficial "turn it off and forget about it" understanding. Recently, 2 references on perioperative management of SCS have been published; however, the first one is written for surgeons addressing the surgical management and the second does not address the myriad anesthetizing locations and the specific implications found in those unique environments $(2,5)$.

In this review, we address the safety concerns of SCS interaction with anesthesia and specialty specific medical equipment. Moreover, we describe SCS implant techniques, as knowledge of SCS location, percutaneous versus laminectomy implant, can determine if neuraxial interventions for anesthesia are possible.

\section{Indications}

Currently, SCS is Food and Drug Administration (FDA) approved for chronic neuropathic pain of the trunk or limbs, radicular pain from failed back surgery syndrome, and pain from complex regional pain syndrome and intractable low back pain (6). However, SCS has been successfully utilized for multiple other indications including chronic intractable angina, peripheral vascular disease, visceral pain postsurgical abdominal pain, and peripheral nerve pain (7). With multiple indications, SCS therapy spans a large patient population potentially requiring $\mathrm{ACT}$ services.

\section{Mechanism of Action}

Neurostimulation uses electrical current applied to neural structures to modulate sensory or autonomic function and improve pain (7). First clinically used in 1967 , the therapy has been refined over the years to reduce complications and improve outcomes (8). The mechanism of action of SCS was initially explained through Melzack and Wall's gate control theory of pain (9). While groundbreaking at the time, this model of pain relief has proven insufficient to fully explain the mechanisms by which SCS modulates neuropathic pain (10). Current animal models would suggest the pain suppressing effects of SCS involves both spinal and supraspinal pathways $(11,12)$. In contrast to the mechanism of action for neuropathic pain, the improvement in painful ischemic conditions results from more than just alteration of nociceptive signals due to ischemia (13). Stimulation appears to positively alter the balance between oxygen supply and demand through reduction in sympathetic tone and resultant vasodilatation of the vasculature $(13,14)$.

\section{Understanding the Device}

In SCS, a wire with electrical contacts (Fig. 1) at the distal portion is placed into the epidural space over the dorsal columns of the spinal cord. The proximal end of the wire is connected to an internal pulse generator (IPG) which delivers energy to the electrodes (15). This device is only implanted after a successful trial documenting reduced pain and improved function $(1,16)$. In appearance, this system resembles a cardiac pacemaker.

Implantation of the device can be achieved in one of 2 ways; through a needle based percutaneous approach to the epidural space or an open laminectomy (17). For percutaneous implant, the needle will enter the epidural space several levels below the anticipated final location of the contacts. For low back and extremity pain coverage, the T8 vertebral level is an appropriate target level with access to the epidural space around the L2/3 interlaminar space. With this approach, the lead would span this distance (T8 to L2/3) within the epidural space. Anchoring of the lead would occur at the point of epidural access (Fig. 1). Laminectomy placement requires an incision directly over the spinal cord target T8 (Fig. 2). The incision will represent the only area where the contacts and lead reside within the epidural space. Regardless of how the lead is placed, the proximal ends are tunneled beneath the skin and connected to the IPG which is placed in a subcutaneous pocket in the flank or abdomen (16).

Currently there are 5 main companies offering SCS systems in the United States: Boston Scientific, Medtronic, Nevro, St. Jude, and Stimwave. While components of each device and the technique for implantation are similar across brands, differences in manufacturing technology affect management recommendations 

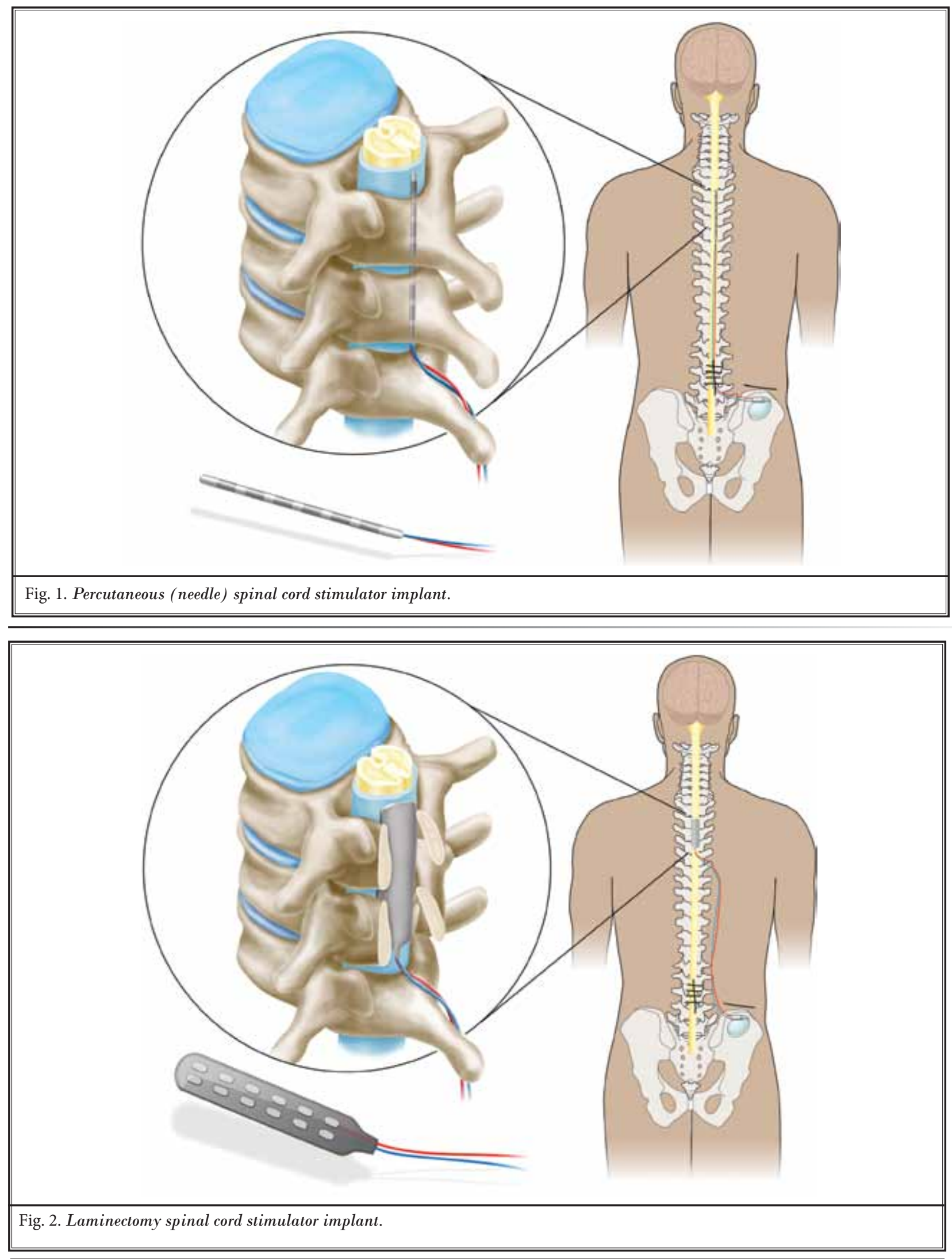


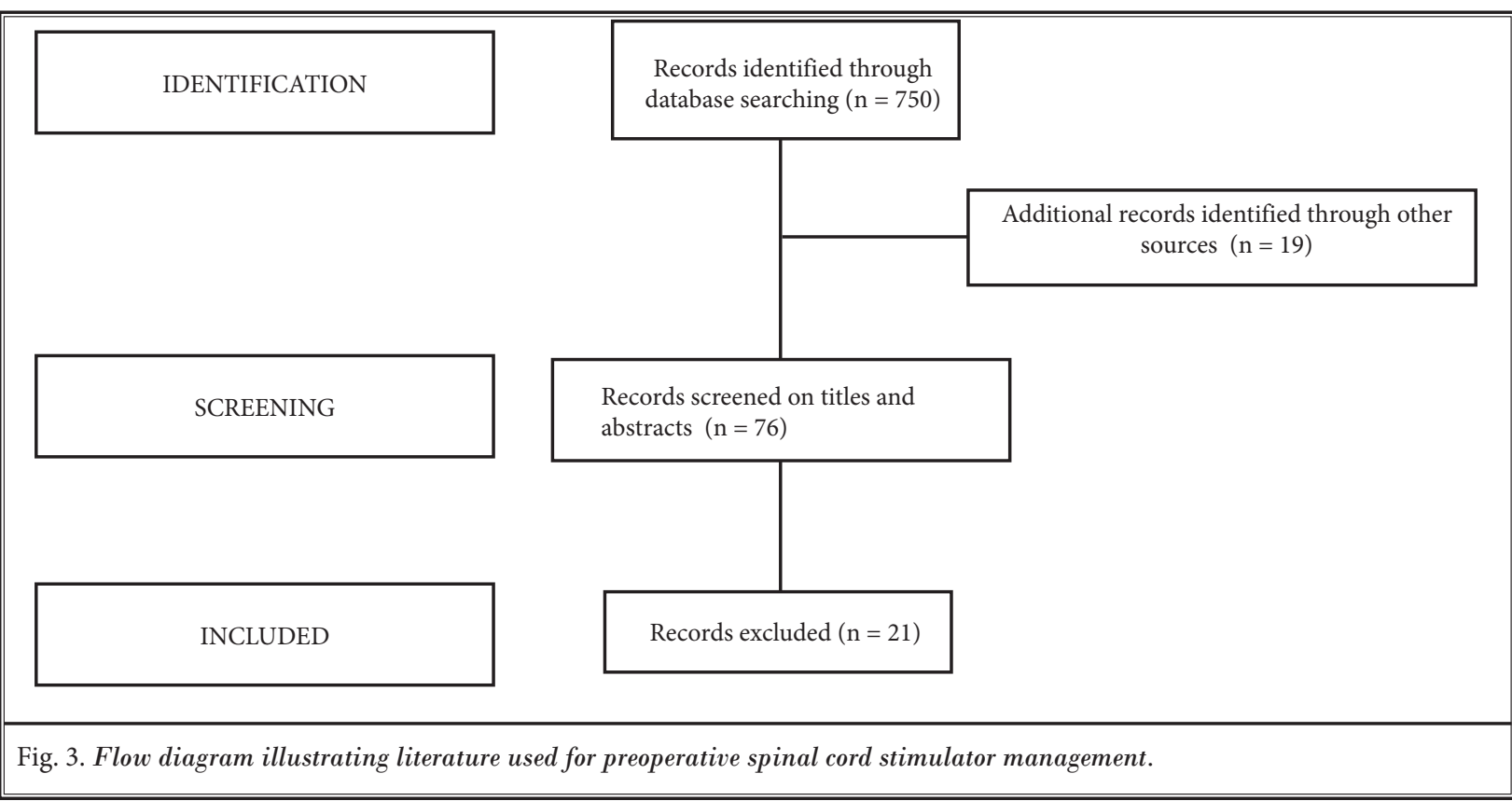

necessitating identification of the specific stimulator brand.

\section{Methods}

\section{Search Strategy}

A systematic search of the literature was conducted in accordance with the Preferred Reporting Items for Systematic Reviews and Meta-Analysis (PRISMA) statement (18). A computerized search was conducted for English articles in print up to April 2016 via PubMed www.ncbi. nlm.nih.gov/pubmed; EMBASE www.embase.com; and Cochrane Library www.thecochranelibrary.com. Search terms included "spinal cord stimulator AND MRI," "spinal cord stimulator AND ECG," "spinal cord stimulator AND implanted cardiac device," "spinal cord stimulator AND electrocautery," and "spinal cord stimulator AND obstetrics". In addition, a search of Google and Google Scholar was performed. Websites of SCS manufactures: https://professional.medtronic.com/pt/neuro/scs/index; www.nevro. com/English/Physicians/Physician-Overview/default; http:// stimwave.com/mobile; www.bostonscientific.com/en-US/ products/spinal-cord-stimulator-systems.html; www.sjm. com/en/patients/chronic-pain were reviewed for product information and perioperative recommendations.

\section{Eligibility Criteria and Study Selection}

Once duplicate results were removed, $\mathrm{MH}$ independently performed review of title and abstract. Included studies reported on (a) observations of implanted device interaction, (b) recommendations of device management, and (c) warnings of device interaction with special equipment.

\section{Data Items and Collection}

All articles with possible relevance were then obtained in full PDF format and reviewed. Relevant articles, which reported on SCS perioperative management, SCS device interactions and complications, and review articles were retained for results (Fig. 3).

\section{Methodologic Quality Assessment}

The quality and validity of each article comprising this analysis was not assessed.

\section{Results}

\section{Operating Room Environment}

While there is little written in the literature about perioperative considerations and management of patients with spinal cord stimulators, multiple clinical scenarios present the potential for risk to patient and/ or device. Most of the information regarding management in the perioperative period can be found in the labeling information provided by the individual manufacturer. That said, with 5 different major companies providing multiple different systems, ready access to relevant information is not always easily available. 


\section{Intraoperative Management}

As a general rule, when a patient presents for an anesthetic, it is recommended that the device be reprogrammed to the lowest possible amplitude and then turned off prior induction of anesthesia (19-23). This ensures if the device is inadvertently turned on, stimulation would be very low and likely be unnoticed. Turning off the device also reduces the risk of accidental reprogramming via electromagnetic interference (EMI) (19-23). It is recommended that the SCS be interrogated post-operatively; however, this does not need to be done in the immediate post-operative setting (22).

\section{ECG}

Electrocardiogram (ECG) interpretation is negatively affected by artifact (24). Typical sources include body movement, tremors, EMI, and implanted electronic devices (24). Under typical clinical scenarios, SCS devices have been shown to interfere with ECG readings resulting in high frequency artifacts in multiple leads (25). Fortunately, this interference is only present when the device is active; therefore, compliance with current SCS operating room recommendations will ensure no ECG artifact from a device programmed to the off mode.

\section{Electrocautery}

Typical monopolar electrocautery creates current which conducts from the electrocautery device through the wound to the grounding pad placed on the patient's body and is then returned to the device (25). This current presents a risk to both the patient and the device (5). A patient, implanted with deep brain stimulator, reported a shocking sensation, described as "lancinating" when exposed to monopolar cautery during a dermatologic procedure (26). Therefore, all 5 SCS companies recommend avoidance of monopolar electrocautery in a patient with SCS (1822) Bipolar electrocautery is recommended if electrocautery is required (2). Different from the monopolar mode, bipolar does not require a grounding pad; the active and return electrodes are on opposite ends of the forceps, limiting energy dispersion (27). If attention is used to keep the device from coming between the 2 electrodes, no harm will come to the device or patient (28).

Despite recommendations, monopolar electrocautery is not always avoidable, as some cases and surgeons require its use. If unavoidable, the device must be interrogated to ensure normal impedances, which confirms no disconnections in the system or damage to the insulating sheath along the lead and electrode. The monopolar electrocautery should be used on the lowest effective setting and the grounding pad should be placed as far away as possible from the SCS and on the contralateral side of the IPG (2). Current operating room recommendations, including turning the device to the lowest setting and then powering off, should be followed. Finally, the SCS must be interrogated after the procedure to ensure proper setting and working order $(2,19,21)$.

\section{Ultrasound and Lithotripsy}

There is no specific scientific literature or case reports when it comes the perioperative management of patients undergoing high output ultrasound or lithotripsy. Current recommendations come from the manufacturers' product manuals (18-22). In general, manufacturers recommend that patients with implanted SCS not undergo high output ultrasound or lithotripsy. Despite this warning, the manufacturers do provide recommendations on device management if the procedure is going to be performed. Recommendations include turning off the stimulator prior to procedures, avoiding focus of the lithotripsy beam within $15 \mathrm{~cm}$ of the SCS, and ensuring functionality at the end of the procedure by powering up the device and then slowly increasing stimulation $(20,22)$.

\section{Pacemaker and Defibrillators}

SCS presents a unique and potentially dangerous interaction with cardiovascular implanted electronic devices (CIEDs), such as permanent pacemakers (PPM) and internal cardiac defibrillator (ICD). Due to the sensing nature of the CIEDs, there is risk that the electrical stimulation from the SCS will interfere with the function of the CIEDs and deliver an inappropriate clinical response (29). In a PPM, there could be suppression of the pacing function, or in the case of an ICD, delivery of an inappropriate defibrillation (29). While manufacturers recommend against the simultaneous use of SCS and CIEDs, there are numerous examples of safe concomitant use in the literature (30-32). Ultimately the decision to implant is between the patient and provider.

Recommendations for concomitant use derived from the literature include obtaining a base line ECG that SCS is known to induce, and placing the CIEDs in bipolar mode if possible $(24,29)$. During the trial and 
implant, interaction between the 2 devices should be elicited. By setting the CIEDs at maximum sensitivity and SCS at maximally tolerated stimulation, any interaction or interference under typical clinical settings would be observed (33). Implantation of the SCS IPG on the contralateral side of the CIED is recommended (34). On a final note, when patients are in lethal arrhythmias, the first consideration is patient survival. External defibrillation and cardioversion can damage the device or induce electrical current in the wire. This is minimized by placing paddles away from the SCS, placing paddles perpendicular to the SCS, and use of the lowest energy appropriate (20).

\section{Obstetric Anesthesia}

As SCS has become employed earlier in the treatment algorithm, patients with chronic pain and coexisting pregnancy are now presenting with implanted SCS requesting analgesia as part of their obstetric services $(1,35)$. Currently there are no studies examining the effects of SCS on human fetal development, and it is likely there never will be (36). As of 2014, there were 8 case reports of a parturient using SCS during pregnancy and receiving neuraxial analgesia for pain associated with labor or caesarian delivery (35). Currently, with the unknown effects of stimulation on fetal development, all the manufacturers recommend deactivation of the device at the time of pregnancy diagnosis (35). Despite no obvious harm, SCS use during pregnancy should only be considered with a careful risk vs. benefit analysis (16). The recommendation to discontinue SCS therapy at time of pregnancy diagnosis continues all the way through delivery. However, SCS has been used without interference with fetal scalp monitoring and external heart rate monitoring using Doppler (36). In some cases, when patients would require potentially teratogenic medications in the absence of SCS, it could be argued for continuing SCS therapy during pregnancy. In fact, one case report suggests that an SCS could be placed for someone who desires to become pregnant and wishes to discontinue certain drugs that would be harmful to the fetus (35).

The choice between an epidural or spinal anesthetic should be chosen based upon the desired anesthetic effect. Epidural solutions are unlikely to cause migration of SCS wires as fibrous deposits form an encapsulated sheath, providing protection along with a well-anchored lead to the supraspinous ligament (37). However, it is this same fibrous tissue that impedes epidural spread and could result in patchy or incomplete analgesia for labor or anesthesia for operative delivery (38). Both spinal and epidural anesthesia can be undertaken at a level below the level of SCS lead entry. Moreover, spinal anesthesia safety has been demonstrated by practitioners who implant SCS devices under spinal anesthesia (39).

While there is potential damage to the SCS system during epidural or spinal placement, this risk is dramatically reduced with prior knowledge of the implant location and technique used. Of the 8 case reports, only half of the authors noted an early anesthesia referral, allowing adequate time for review of records and anesthetic planning (35). During placement, strict sterile technique must be employed as well as special attention to securing the catheter to minimize dislodgment and infection risk (40). If placement of a needle were within adjacent vertebral body levels of the known SCS lead location, it would likely be safer to defer regional techniques in favor of intravenous patient controlled analgesia for labor and general anesthesia for caesarian delivery (41).

It is the reviewers' recommendation that an early referral is made to the obstetric anesthesia service for delivery planning. In this evaluation, prior radiographs and implant records should be obtained to identify the location of the epidural leads and method of implant. The anesthetic plan should be developed and coordinated with all persons likely to be involved in the patient's care. It is also the reviewers' recommendation that a parturient not requesting labor analgesia or expecting the need for anesthesia be referred in case of emergency caesarian delivery.

\section{Acute Pain}

Regional and epidural analgesia have been demonstrated to reduce morbidity, improve perioperative pain control, and improve patient satisfaction $(42,43)$. However, in patients with indwelling SCS, neuraxial analgesia and regional techniques might not be offered due to concerns of injury to the patient and/or device or potential failure of the block. One case report exists of failed epidural analgesia for acute pain management in a patient with in situ high thoracic SCS for chronic pericarditis pain (44). Otherwise, the literature is currently silent on the concomitant use of neuraxial analgesia for acute pain management in patients with implanted SCS. Extrapolation of the obstetric literature where neuraxial analgesia has been used in patients with indwelling SCS would suggest its feasibility $(35,36)$. However, neuraxial access for most obstetric anesthesia and analgesia involves the lower lumber region. Acute 
pain management will often require placement of an epidural needle and catheter at the appropriate dermatomal level to ensure analgesic coverage of the surgical site, typically T6-T10. These spinal levels are also the most common levels for SCS lead placement for the management of low back and leg pain (45). While there is no literature to rely on, it would be the authors' opinion that in this case, the risk benefit ratio is not in favor of placement.

Even if an epidural catheter is placed, there is no guarantee that desired pain relief will occur due to the well-known development of fibrosis within the epidural space $(37,38)$. This epidural fibrosis may limit the spread and penetration of epidural administered medications thereby limiting effectiveness (38).

Currently, the only recommendations would be to apply caution when considering an elective neuraxial technique in patients with implanted SCS. The proposed site of the procedure relative to the known or presumed placement of the implanted device should be considered. Moreover, the efficacy of the technique might be reduced due to poor epidural spread.

\section{Non-Operating Room Anesthesia}

Non-operating room anesthesia (NORA) refers to providing anesthesia or sedation for prolonged or painful procedures outside the operating room (46). As a result of improved technology and technical advances, the frequency of these procedures is increasing (47). In some centers, up to $30 \%$ of all anesthetics are provided outside of the operating room (48). Often these procedures are performed in a special suite specifically suited to the needs of the intervention. In these highly technical environments, SCS can have significant interaction with the equipment and pose significant risk to the device and patient as well as hamper the procedure.

\section{MRI}

As noted earlier, chronic pain patients have higher than average health care utilization (3). Moreover, calculated rates of expected need for spine MRI in a patient with implanted SCS over the next 5 years is $82 \%$ - $84 \%$ (49). Unfortunately, recommendations on MRI compatibility show the biggest differences between SCS manufacturers and current systems available on the market. Unfortunately, failure to follow these recommendations will likely result in the greatest morbidity amongst all possible SCS medical device interactions. Prior to 2013, there was no SCS system which carried full-body conditional labeling; this changed with
Medtronic's SureScan technology (50). Since then, Boston Scientific's Precision, Stimwave's Freedom, and most recently St. Jude's Proclaim are all full-body conditional MRI complatible $(23,51,52)$. The remaining company, Nevro, has conditional labeling for specific body parts under certain conditions (53).

Implanted SCS poses a risk to the patient undergoing MRI from thermal injury across all parts of the device (2). The induction of current into the device comes from components of the MRI environment (55). The first component of the MRI environment to cause potential harm is the large magnet, which aligns protons within the tissues. This magnet acts on any ferromagnetic materials with a SCS. The second component of the MRI environment which can pose hazard is the pulsed radiofrequency (RF) fields, which excites the protons. Finally, there are pulsed magnetic fields used to localize the signal generated by the RF pulses (54). These 3 forces can result in heating of the spinal cord lead, magnetic pull on the device, damage to the device, and unwanted stimulation. Heating of the lead is an issue given its proximity to neural structures. Magnetic pull could result in torque or rotation movement of the device with potential destruction of surrounding tissues. As the device potentially escapes from its implanted pocket, the object may accelerate into the bore becoming a missile, exposing additional risk to the patient, and a new risk to the health care team and the machine itself $(47,56)$.

In order to prospectively assess the risk between MRI and SCS, in 2007, De Andres et al (55) attempted to develop a protocol for scanning patients with implanted SCS systems. In this study, $31 \mathrm{MRI}$ scans were performed with a total of 7 events reported during the scan. Five of the patients noted their typical pattern of stimulation during the MRI despite the fact that the SCS was turned off. Two patients reported the sensation increased temperature in the area of the generator, however; there were no obvious burns on inspection. SCS evaluation after MRI revealed 4 patients had experienced significant changes to their programming. In 2 patients, telemetry between IPG and patient programmer failed, necessitating replacement of the IPG. In 2015, the American Society of Anesthesiologists (ASA) updated its practice advisory related to anesthetic care for patients in the MRI environment (57). It acknowledged that newer cardiac systems were now MRI compatible but did not discuss the SCS compatible systems. It did recommend education regarding SCS and its potential interaction with the MRI environment (57). 
Current recommendations would include that the ACT first identify the device manufacturer and model. With this information, the specific device manual will clearly outline what type of MRI and under what conditions the device can be scanned. Another fast reference used by the technologist in our institution is mrisafety. com (58). As of this writing, all manufacturers offer a SCS system, which is conditionally labeled for MR scanning of the head (Table 1). If a patient presents for elective MRI and does not have documentation explicitly outlining MRI compatibility, then the scan should be delayed until adequate documentation can be obtained. In the event a patient requires emergent MRI and documentation is unavailable, then the benefits of proceeding must be weighed against the risks and consideration given to alternative imagining modalities.

\section{Radiation Medicine}

Radiotherapy (RT) utilizes high dose ionizing radia- tion focused at a tumor site for potential cure or palliation of malignancies. It has been reported that RT is utilized in approximately $60 \%$ of patients at some point during cancer treatment (59). Unfortunately, this potentially lifesaving therapy can also damage implanted devices such as PPM, ICD, or SCS systems (60). Damage to the device as a result of ionizing radiation is cumulative, permanent, and can lead to device failure (60). The severity of the damage is dependent on the radiation type, total dose, and type of device. Unfortunately, the damage can occur whether the device is in the on or off mode (61).

Given the increasing use of implanted devices, recommendations have been developed to safely deliver radiation therapy in patients with pre-existing devices (62). However, to date there are no studies that investigate the impact of RT on SCS systems specifically. Instead, current recommendations are based on case reports and extrapolation of recommendations made for CIEDs (62). Walsh et al reported a case of a patient

Table 1. Summary of spinal cord stimulator perioperative management recommendations.

\begin{tabular}{|c|c|c|c|}
\hline Case & Preoperative Considerations & Intraoperative Considerations & $\begin{array}{l}\text { Post-operative } \\
\text { Considerations }\end{array}$ \\
\hline Electrocautery & $\begin{array}{l}\text { Program SCS to lowest amplitude; turn } \\
\text { off prior to case }(20,22)\end{array}$ & $\begin{array}{l}\text { Use bipolar cautery. } \\
\text { If monopolar cautery required, use } \\
\text { lowest effective setting and place } \\
\text { grounding pad a far from SCS as } \\
\text { possible (2) }\end{array}$ & $\begin{array}{l}\text { Interrogation for device } \\
\text { damage/failure (19-23) }\end{array}$ \\
\hline Ultrasound /Lithotripsy & $\begin{array}{l}\text { Turn off the stimulator prior to procedure } \\
(20,22)\end{array}$ & $\begin{array}{l}\text { Avoid focus of the lithotripsy beam } \\
\text { within } 15 \mathrm{~cm} \text { of the SCS }(20,22)\end{array}$ & $\begin{array}{l}\text { Interrogation for device } \\
\text { damage/failure }(20,22)\end{array}$ \\
\hline Pacemakers /Defibrillators & $\begin{array}{l}\text { Obtain baseline EKG to rule out new } \\
\text { artifact } \\
\text { Set PPM/CEID and SCS to bipolar mode } \\
(24,29)\end{array}$ & $\begin{array}{l}\text { Should lethal arrhythmia occur, place } \\
\text { paddles away and perpendicular } \\
\text { to SCS and use lowest appropriate } \\
\text { energy (20) }\end{array}$ & $\begin{array}{l}\text { Interrogation for device } \\
\text { damage/failure }(24,29)\end{array}$ \\
\hline Obstetrics & $\begin{array}{l}\text { Infer lead location based on patient scars, } \\
\text { see figures } 1 \text { and } 2\end{array}$ & Proceed as clinically appropriate & \\
\hline Acute Pain & $\begin{array}{l}\text { Consider SCS lead location relative to site } \\
\text { of procedure }\end{array}$ & $\begin{array}{l}\text { Consider efficacy given possibility of } \\
\text { epidural fibrosis, proceed as clinically } \\
\text { appropriate (38) }\end{array}$ & \\
\hline MRI & $\begin{array}{l}\text { Determine brand and model of SCS } \\
(23,50-53)\end{array}$ & $\begin{array}{l}\text { Determine brand and model of SCS } \\
(23,50-53)\end{array}$ & \\
\hline Radiation Therapy & $\begin{array}{l}\text { Total dose limit should be less than } 5 \mathrm{~Gy} \\
\text { and the pulse generator should be greater } \\
\text { than } 1 \text { centimeter outside of the direct } \\
\text { beam }(62)\end{array}$ & $\begin{array}{l}\text { Undergo treatment with device in off } \\
\text { position }(61)\end{array}$ & $\begin{array}{l}\text { Interrogation for device } \\
\text { damage or failure (61) }\end{array}$ \\
\hline CT & $\begin{array}{l}\text { The SCS device should be turned off } \\
\text { during the scan (63) }\end{array}$ & $\begin{array}{l}\text { The CT scan should use lowest dose } \\
\text { necessary to obtain the images ( } 63 \text { ) }\end{array}$ & $\begin{array}{l}\text { Interrogation for proper } \\
\text { function }(63)\end{array}$ \\
\hline $\begin{array}{l}\text { Microwave Ablation/ } \\
\text { Diagnostic Ultrasound }\end{array}$ & $\begin{array}{l}\text { Potential for heating of the lead, electrode } \\
\text { or IPG with microwave ablation (20) } \\
\text { Potential for disruption of connections } \\
\text { with ultrasound (20) }\end{array}$ & $\begin{array}{l}\text { If feasible, maintain consciousness to } \\
\text { report heating/abnormal sensations } \\
\text { (20) }\end{array}$ & $\begin{array}{l}\text { Interrogation for disruption } \\
\text { or other damage (20) }\end{array}$ \\
\hline
\end{tabular}


who required adjuvant RT for breast cancer with a SCS in-situ (61). In this report, they recommended the total dose limit should be less than 5 gray (Gy) and the pulse generator to be greater than one centimeter outside of the direct beam. If the pulse generator falls within the treatment beam, it should be removed prior to treatment and replaced once completed. The patient should undergo treatment with the device in the off position and dosimetry measurements should be taken at the skin surface on 3 separate occasions (61). Current manufacturers' recommendations can be generalized between companies and are similar to recommendations proposed by Walsh with the addition of device shielding when the target is near the IPG (20).

\section{Computed Tomography}

Computed tomography (CT) scanning is the preferred method of diagnostic imaging in patients with SCS and typically provides adequate information in a majority of cases (5). Recently, concerns with the use of CT scanning in patients with implanted devices including SCS, DBS, and CIEDs have been reported (63). Specifically, high levels of radiation have resulted in patients reporting a shocking sensation during scanning and device lead malfunction (64). While the FDA considers the risk extremely low, recommendations have been made to limit this risk (63). Regarding the device, it is recommended that the device be turned off during the scan and once complete the patient should turn the device back on to check for proper function. The CT scan should use lowest dose necessary to obtain the images and should avoid excessive scanning through the body area with the implanted device (63).

\section{Microwave Ablation \& Diagnostic Ultrasound}

Microwave ablation is typically performed using CT guidance, which as discussed can be a potential source of device interaction and patient harm (63). The safe use of concomitant microwave ablation and SCS has not been established. There is potential for induction of currents with resultant heating of the lead or IPG (20). The only recommendation that could be made would be to address the risk/benefit ratio on a caseby-case basis. If possible, keeping the patient awake so that they can report any abnormal sensations would be recommended, understanding this might not be possible with solid organ ablative procedures. Care should be exercised while performing diagnostic ultrasound in patients with SCS. It has been noted that the back and forth motion of the ultrasound transducer directly over the system can lead to potential disruption of the lead/ extension connections and/or loosening of sutures (20).

\section{Discussion}

SCS have been established as effective treatment for a variety of chronic and ischemic pain syndromes. As a result, the ACT must be prepared to manage SCS in the perioperative period, both in and outside the operating room. For a patient undergoing a general or MAC anesthetic without medical equipment interference, the recommendation would be to program the device to the lowest amplitude and then turn the device off prior to starting the case. Monopolar electrocautery should be avoided in place of bipolar cautery. If not possible, a grounding pad should be placed as far away from the SCS. While concomitant use of SCS and CIED is not an absolute contraindication, it is a risk/benefit decision between the physician and patient. Care should be taken to implant the SCS as far away from the CIED as possible.

SCS is a viable choice for women of child-bearing age. While a number of unique challenges face the obstetrician and anesthesiologist in managing a pregnant woman with an SCS, neuraxial analgesia need not be denied to the patient. It is recommended and ideal that a parturient with implanted SCS meets with the ACT prior to labor to review x-rays and records so that an anesthetic plan may be developed prior to presenting for delivery. If this is not feasible, the ACT should have an understanding of SCS placement and be able to infer lead location based upon patient scars. This understanding naturally applies to acute pain procedures as well.

MRI conditional labeling is the most differentiating feature of the SCS models and manufacturers. Beyond the full body labeling from Medtronic, Boston Scientific, and Stimwave, each company has specific labeling restrictions. The best recommendation is to first determine the brand and model SCS, and then obtain the appropriate manual to review MRI compatibility.

\section{Conclusion}

SCS is an effective tool in the management of many different chronic pain conditions. This efficacy means that it will be utilized with more frequency and sooner in the treatment algorithm. As patients present for anesthetic care, the ACT will need to have a comprehensive understanding of the device and how it interacts with various other technologies utilized in the care of patients. 


\section{References}

1. Deer T. Spinal cord stimulation for the treatment of chronic pain. Pain Medicine News 2010 July; 1-8.

2. Walsh KM, Machado AG, Krishnaney AA. Spinal cord stimulation: A review of the safety literature and proposal for perioperative evaluation and management. Spine ] 2015; 15:1864-1869.

3. Institute of Medicine (US) Committee on Advancing Pain Research, Care, and Education. Relieving Pain in America: A Blueprint for Transforming Prevention, Care, Education, and Research. National Academies Press, Washington DC, 2011.

4. Grider JS, Brown RE, Colclough GW. Perioperative management of patients with an intrathecal drug delivery system for chronic pain. Anesth Analg 2008; 107:1393-1396.

5. Ghaly RF, Tverdohleb T, Candido KD, Knezevic NN. Do we need to establish guidelines for patients with neuromodulation implantable devices, including spinal cord stimulators undergoing nonspinal surgeries? Surg Neurol Int 2016; 7:18.

6. Deer TR. Current and future trends in spinal cord stimulation for chronic pain. Curr Pain Headache Rep 2001; 5:503-509.

7. Reig E, Abejon D. Spinal cord stimulation: A 20-year retrospective analysis in 260 patients. Neuromodulation 2009; 12:232-239.

8. Shealy CN, Mortimer JT, Reswick JB. Electrical inhibition of pain by stimulation of the dorsal columns: Preliminary clinical report. Anesth Analg 1967; 46:489-491.

9. Melzack R, Wall PD. Pain mechanisms: A new theory. Science 1965; 150:971-979.

10. Linderoth B, Foreman RD. Mechanisms of spinal cord stimulation in painful syndromes: Role of animal models. Pain Med 2006; 7:S14-S16.

11. Barchini J, Tchachaghian S, Shamaa F, Jabbur SJ, Meyerson BA, Song Z, Linderoth B, Saadé NE. Spinal segmental and supraspinal mechanisms underlying the pain-relieving effects of spinal cord stimulation: An experimental study in a rat model of neuropathy. Neuroscience 2012; 215:196-208.

12. Saadé NE, Jabbur SJ. Nociceptive behavior in animal models for peripheral neuropathy: Spinal and supraspinal mechanisms. Prog Neurobiol 2008; 86:22-47.

13. Linderoth B, Foreman RD. Physiology of spinal cord stimulation: Review and update. Neuromodulation 1999; 2:150-164.
14. Naoum JJ, Arbid EJ. Spinal cord stimulation for chronic limb ischemia. Methodist DeBakey Cardiovasc J 2013; 9:99-102.

15. De Ridder D, Plazier M, Kamerling N Menovsky T, Vanneste S. Burst spinal cord stimulation for limb and back pain. World Neurosurg 2013; 80:642-649.

16. Raff M, Melvill R, Coetzee G, Smuts J. Spinal cord stimulation for the management of pain: Recommendations for best clinical practice. S Afr Med J 2013; 103:423-430.

17. Deer T, Pope J, Hayek S, Narouze S, Patil $P$, Foreman R, Sharan A, Levy R. Neurostimulation for the treatment of axial back pain: A review of mechanisms, techniques, outcomes, and future advances. Neuromodulation 2014; 17:52-68.

18. Liberati A, Altman DG, Tetzlaff J, Mulrow C, Gøtzsche PC, loannidis JP, Clarke M, Devereaux PJ, Kleijnen J, Moher D. The PRISMA statement for reporting systematic reviews and meta-analyses of studies that evaluate health care interventions: Explanation and elaboration. Ann Intern Med 2009; 151:W65-W94.

19. Nevro. Physician implant data: www. accessdata.fda.gov/cdrh_docs/pdf 13 / P130022d.pdf (accessed March 15, 2016).

20. Medtronic Pain Therapy. http://manuals.medtronic.com/wcm/groups/mdtcom_sg/@emanuals/@era/@neuro/ documents/documents/contrib_199974. pdf (accessed March 15, 2016).

21. St. Jude Medical. Power over your pain. www.poweroveryourpain.com/safety/SC SSafety (accessed March 15, 2016).

22. Precision Spectra ${ }^{\mathrm{TM}}$ Spinal Cord Stimulator System. http://hcp.controlyourpain. com/scs-safety-information/precisionspectra (accessed March 15, 2016).

23. Stimwave Technologies. Freedom spinal cord stimulator trial kit manual.

http://stimwave.com/mobile/wp-content/ uploads/2016/01/LA-FR8A-1-869-TrialInstructions-for-Use.pdf

(accessed June 2, 2016).

24. Khan IA. Differential electrocardiographic artifact from implanted thalamic stimulator. Int J Cardiol 2004; 96:285-286.

25. Siddiqui MA, Khan IA. Differential electrocardiographic artifact from implanted spinal cord stimulator. Int ] Cardiol 2003; 87:307-309.

26. Voutsalath MA, Bichakjian CK, Pelosi F, Blum D, Johnson TM, Farrehi PM. Electrosurgery and implantable electronic devices: Review and implications for office-based procedures. Dermatol Surg
2011; 37:889-899.

27. Weaver J, Kim SJ, Lee MH, Torres A. Cutaneous electrosurgery in a patient with a deep brain stimulator. Dermatol Surg 1999; 25:415-417.

28. Massarweh NN, Cosgriff N, Slakey DP. Electrosurgery: History, principles, and current and future uses. J Am Coll Surg 2006; 202:520-530.

29. Ooi YC, Falowski S, Wang D, Jallo J, Ho RT, Sharan A. Simultaneous use of neurostimulators in patients with a preexisting cardiovascular implantable electronic device. Neuromodulation 2011; 14:20-25; discussion 25-26.

30. Kosharskyy B, Rozen D. Feasibility of spinal cord stimulation in a patient with a cardiac pacemaker. Pain Physician 2006; 9:249-251.

31. Ekre O, Borjesson M, Edvardsson N, Eliasson T, Mannheimer C. Feasibility of spinal cord stimulation in angina pectoris in patients with chronic pacemaker treatment for cardiac arrhythmias. Pacing Clin Electrophysiol 2003; 26:2134-2141.

32. Hoelzer BC, Burgher AH, Huntoon MA. Thoracic spinal cord stimulation for post-ablation cardiac pain in a patient with permanent pacemaker. Pain Pract 2008; 8:110-113.

33. Monahan K, Casavant D, Rasmussen C, Hallet N. Combined use of a true-bipolar sensing implantable cardioverter defibrillator in a patient having a prior implantable spinal cord stimulator for intractable pain. Pacing Clin Electrophysiol 1998; 21:2669-2672.

34. lyer R, Gnanadurai TV, Forsey P. Management of cardiac pacemaker in a patient with spinal cord stimulator implant. Pain 1998; 74:333-335.

35. Patel S, Das S, Stedman RB. Urgent cesarean section in a patient with a spinal cord stimulator: Implications for surgery and anesthesia. Ochsner J 2014; 14:131-134.

36. Bernardini DJ, Pratt SD, Takoudes TC, Simopoulos TT. Spinal cord stimulation and the pregnant patient-specific considerations for management: A case series and review of the literature. Neuromodulation 2010; 13:270-274.

37. Loge D, Devulder JE, De Coster O, De Colvenaer L, Mortier E. The epidural fibrous sheath: A guide for the replacement of a spinal cord stimulation electrode. Reg Anesth Pain Med 2002; 27:353-356.

38. Helm S, Racz GB, Gerdesmeyer L, Justiz 
R, Hayek SM, Kaplan ED, El Terany MA, Knezevic N. Percutaneous and endoscopic adhesiolysis in managing low back and lower extremity pain: A systematic review and meta-analysis. Pain Physician 2016; 19:E245-E281.

39. Bedder MD, Bedder HF. Spinal cord stimulation surgical technique for the nonsurgically trained. Neuromodulation 2009; 12:1-19.

40. Deer TR, Stewart CD. Complications of spinal cord stimulation: Identification, treatment, and prevention. Pain Med 2008; 9:S93-S101.

41. Halpern SH, Muir H, Breen TW, Campbell DC, Barrett J, Liston R, Blanchard JW. A multicenter randomized controlled trial comparing patient-controlled epidural with intravenous analgesia for pain relief in labor. Anesth Analg 2004; 99:1532-1538.

42. Rodgers A, Walker N, Schug S, McKee A, Kehlet $H$, van Zundert A, Sage D, Futter M, Saville G, Clark T, MacMahon S. Reduction of postoperative mortality and morbidity with epidural or spinal anaesthesia: Results from overview of randomised trials. BM] 2000; 321:1493.

43. Beattie WS, Badner NH, Choi P. Epidural analgesia reduces postoperative myocardial infarction: A meta-analysis. Anesth Analg 2001; 93:853-858.

44. Harned ME, Baker M, Sloan P. Epidural analgesia fails to provide acute pain relief in patient with implanted spinal cord stimulator for chronic pain. American Society of Anesthesiologist Annual Meeting. October 2015. San Diego, California.

45. Holsheimer J. Which neuronal elements are activated directly by spinal cord stimulation. Neuromodulation 2002; 5:25-31.

46. Metzner J, Domino KB. Risks of anesthesia or sedation outside the operating room: The role of the anesthesia care provider. Curr Opin Anaesthesiol 2010; 23:523-531.
47. Youn AM, Ko YK, Kim YH. Anesthesia and sedation outside of the operating room. Korean J Anesthesiol 2015; 68:323-331.

48. Souter KJ, Davies JM. Diversification and specialization in anesthesia outside the operating room. Curr Opin Anaesthesio 2012; 25:450-452.

49. Desai MJ, Hargens LM, Breitenfeldt MD, Doth AH, Ryan MP, Gunnarsson C, Safriel $Y$. The rate of magnetic resonance imaging in patients with spinal cord stimulation. Spine 2015; 40:E531-E537.

50. Medtronic. MRI guidelines for Medtronic neurostimulation systems for chronic pain. http://manuals.medtronic.com/ wcm/groups/mdtcom_sg/@emanuals/@era/@neuro/documents/documents/contrib_171957.pdf (accessed April 2, 2016).

51. Boston Scientific. Precision Montage MRI spinal cord stimulator system. www.bostonscientific.com/content/dam/ Manuals/us/current-rev-en/91053659-01_ RevC_Precision_Montage_MRI_Information_for_Prescribers_DFU_en-USA_S.pdf (accessed June 1, 2016).

52. Nevro. MRI guidelines for the Senza system. http://nevro-wp.element68. com/wp-content/uploads/11096-Rev.-ASenza-System-1-5T-and-3T-MRI-Guidelines.pdf (accessed April 2, 2016).

53. St. Jude Medical. Protégé MRI SCS system for chronic pain. https://professional.sjm.com/therapies/protege-mri/ safety-info (accessed April 2, 2016)

54. Boston Scientific. ImageReady MRI guidelines. http://hcp.controlyourpain. com/hcp/assets/File/90894518_ImagerReady\%20MRI\%20Guidelines.pdf (accessed April 2, 2016).

55. De Andres J, Valia JC, Cerda-Olmedo G, Quiroz C, Villanueva V, Martinez-Sanjuan $V$, de Leon-Casasola O. Magnetic resonance imaging in patients with spinal neurostimulation systems. Anesthesiology 2007; 106:779-786.
56. Mutter UM, Bellut D, Porchet F, Schuknecht B. Spinal magnetic resonance imaging with reduced specific absorption rate in patients harbouring a spinal cord stimulation device - A single-centre prospective study analysing safety, tolerability and image quality. Acta Neurochir 2013; 155:2327-2332.

57. Practice advisory on anesthetic care for magnetic resonance imaging: An updated report by the American Society of Anesthesiologists task force on anesthetic care for magnetic resonance imaging. Anesthesiology 2015; 122:495-520.

58. MRIsafety.com (accessed April 15, 2016).

59. Delaney G, Jacob S, Featherstone C, Barton $\mathrm{M}$. The role of radiotherapy in cancer treatment: Estimating optimal utilization from a review of evidencebased clinical guidelines. Cancer 2005; 104:1129-1137.

6o. Solan AN, Solan MJ, Bednarz G, Goodkin MB. Treatment of patients with cardiac pacemakers and implantable cardioverter-defibrillators during radiotherapy. Int J Radiat Oncol Biol Phys 2004; 59:897-904.

61. Walsh L, Guha D, Purdie TG, Bedard P, Easson A, Liu FF, Hodaie M. Spinal cord stimulators and radiotherapy: First case report and practice guidelines. Radiat Oncol 2011; 6:143.

62. Gelblum DY, Amols H. Implanted cardiac defibrillator care in radiation oncology patient population. Int J Radiat Oncol Biol Phys 2009; 73:1525-1531.

63. FDA. Electromagnetic compatibility. http://www.fda.gov/radiation-emittingproducts/radiationsafety/electromagneticcompatibilityemc/default.htm (accessed April 5, 2016).

64. Porres JM, Cerezuela JL, Luque O, Marco P. Computed tomography scan and ICD interaction. Case Rep Med 2009; 2009:189429. 
